\title{
Author Correction: Intermediate degrees of synergistic pleiotropy drive adaptive evolution in ecological time
}

Léa Frachon, Cyril Libourel, Romain Villoutreix, Sébastien Carrère, Cédric Glorieux, Carine Huard-Chauveau, Miguel Navascués, Laurène Gay, Renaud Vitalis, Etienne Baron, Laurent Amsellem, Olivier Bouchez, Marie Vidal, Valérie Le Corre, Dominique Roby, Joy Bergelson and Fabrice Roux

Correction to: Nature Ecology \& Evolution 1, 1551-1561 (2017); published online 4 September 2017.

In the version of this Article previously published, there was a typographical error (' 4 ' instead of ' 2 ') in the equations relating $F_{\mathrm{ST}}$ and effective population size $\left(N_{\mathrm{e}}\right)$ in the Methods section 'Genome-wide scan for selection based on temporal differentiation'. The correct equations are given below.

$$
\begin{gathered}
F_{\mathrm{ST}} \approx \frac{T}{T+2}=\frac{\tau}{\tau+2 N_{\mathrm{e}}} \\
\widehat{N}_{\mathrm{e}}=\frac{\tau\left(1-\widehat{\bar{F}}_{\mathrm{ST}}\right)}{2 \widehat{\bar{F}}_{\mathrm{ST}}}
\end{gathered}
$$

All the calculations and results presented in the Article were obtained using these correct equations and need no amendment. The conclusions of our study are therefore in no way affected. 